\title{
N92-14089
}

\section{AN ANALYSIS OF THE HUBBLE SPACE TELESCOPE FINE GUIDANCE SENSOR FINE LOCK MODE*}

\author{
L. G. Taff \\ Space Telescope Science Institute \\ 3700 San Martin Drive \\ Baltimore, MD 21218
}

\begin{abstract}
There are two guiding modes of the Hubble Space Telescope used for the acquisition of astronomI data by one of its six scientific instruments. The more precise one is called Fine Lock. Command 1 control problems in the on-board electronics has limited Fine Lock to brighter stars, $V<13.0$ $\mathrm{g}$, instead of fulfilling its goal of $V=14.5 \mathrm{mag}$. Consequently, the less precise guiding mode of arse Track ( $\sim 0$ milli-arc seconds) has to be used fairly frequently. Indeed, almost half of the estial hemisphere has stars too faint to support a Fine Lock guidance mode. Hence, some of the entific observations to have been made with the Hubble Space Telescope will be compromised. In s paper I report on the only realistic or extensive simulations of the Fine Lock guidance mode. e theoretical analysis underlying the Monte Carlo experiments and the numerical computations arly show both that the control electronics are severely under-engineered and how to adjust the ious control parameters to successfully extend Fine Lock guiding performance back to $V=14.0$ ig and sometimes beyond.
\end{abstract}

\section{INTRODUCTION}

This paper is complementary to Taff (1990a) in which the Coarse Track mode of the Hubble ace Telescope Fine Guidance Sensors was analyzed. The motivation for that paper was the desire considerably shorten Guide Star acquisition times-thereby significantly enhancing the efficiency Hubble Space Telescope operations-without a loss of scientific information. The key issue was a listic estimate of the Coarse Track guiding mode pointing stability, methods to improve upon it, d whether or not this level of pointing stability would compromise the scientific content of some ibble Space Telescope observations.

The real Optical Telescope Assembly-because of manufacturing errors, wavefront calibration alysis errors, and the tilt and decenter of the secondary mirror-is seriously degrading the guiding rformance of the Fine Guidance Sensors. Instead of a 17-20 milli-arc second Coarse Track pointing ecision, we more typically experience a 40 milli-arc second (mas) pointing instability. Instead of Fine .ck to $V=14.5 \mathrm{mag}$, operationally successful Fine Lock stops at $V=13.0 \mathrm{mag}$. The implication a thirteenth magnitude Fine Lock limit is, in effect, no Hubble Space Telescope observations, with ne Lock, beyond a galactic latitude limit of $|b|=30^{\circ}$. This means excluding half of the celestial misphere and most of the extra-galactic part of the sky. Thus, a complete investigation of the Fine ick algorithm was undertaken in an attempt to rapidly make the maximum improvements. This per summarizes that effort.

The next section of the main text addresses the limiting magnitude issue in more depth. With a zarer understanding of the importance of the Fine Lock limiting magnitude, I then briefly review e Fine Guidance Sensor electro-optical system $(\S 3)$ and the principles of Fine Guidance Sensor idance operation $(\$ 4)$. Section 5 summarizes a theoretical analysis of Fine Lock and extensive

\footnotetext{
"Based on observations with the NASA/ESA Hubble Space Telescope, obtained at the Space lescope Science Institute, which is operated by the Association of Universities for Research in stronomy, Inc., under NASA contract NAS5-26555.
} 
computer simulations of Fine Lock. The latter are based on empirical Fine Guidance Sensor Transfer Functions.

\section{GUIDE STAR PHOTOMETRIC STATISTICS}

The operational problem faced during the latter half of 1990 was the unreliability of the Fine Lock process of the Hubble Space Telescope (HST) Fine Guidance Sensors (FGSs) operating under the Pointing Control System. ${ }^{1}$ Without now going into the detail which will be provided in Section 4 , the Fine Lock mode is the ultimate in the control of the spacecraft. The successful attainment and maintenance of this state is crucial for reaching some of the scientific goals of the HST mission for only with the pointing stability of Fine Lock ( $~ 7$ milli-arc seconds) can some of the instrumental modes function optimally. This became even more important when the spherical aberration in the primary mirror degraded the pointing stability of the Coarse Track mode by a factor of two, from $\sim 20$ milli-arc seconds to $\sim 40$ mas. The Guide Star Catalog, a catalog of stars from which guiding targets for the FGSs are to be chosen, typically reaches $V=14.5 \mathrm{mag}$ for this was the specified limit of a successfully achieved and maintained Fine Lock state. Unfortunately, the real performance of the hardware and Perkin-Elmer Corp.'s utilization of it limited a stable Fine Lock state to $V<13.0$ mag and Fine Lock was routinely unobtainable beyond $V=13.0$ mag. These poor results, the improvement of which is the main subject of this paper, would have placed severe limitations on the scientific operational capabilities of the HST.

To understand how a significantly brighter limiting magnitude for Guide Stars affects the scientific mission of the HST we must understand how the Guide Star Catalog was created. The stellar density goals were $\sim 500$ stars per square degree, uniformly over the entire celestial sphere, to a fixed limiting magnitude. Our location in the Milky Way, the spiral nature of the Galaxy, and the underlying galactic luminosity distribution all conspire to prevent one from attaining this goal. As the constructors of the Guide Star Catalog moved towards the galactic poles they were forced to go fainter and fainter to maintain a constant stellar density. Not knowing, in advance, where the General Observers who would use the HST might want to point it, allowance had to be made for all-sky coverage with a uniform areal density. The apparent magnitude distributions for three galactic latitudes are shown in Fig. 1 wherein the faintward shift at higher galactic latitudes can be seen. However, since the Guide Star Catalog is not a complete catalog, the limiting magnitude does not decrease as rapidly with increasing absolute value of galactic latitude as the true stellar density decreases. Figure 2 provides an integrated (over galactic latitude) apparent magnitude distribution for the entire Guide Star Catalog. There is a displacement of $\sim 0.6$ mag between the two celestial hemispheres because of a color term between the $\sim V$ sensitivity of the northern hemisphere Schmidt plates and the $\sim J$ sensitivity of the southern hemisphere Schmidt plates used to construct the Guide Star Catalog.

To achieve Fine Lock in two of the three FGSs, which is what is necessary for the Pointing Control System to be satisfied, we require two stars brighter than the limiting magnitude of Fine Lock guidance. If, instead of being able to avail ourselves of the full $V=14.5 \mathrm{mag}$ limit of the Guide Star Catalog we are forced to retreat to $V=13.0 \mathrm{mag}$, then the $a$ priori probability of being able to achieve Fine Lock - solely because of the lack of suitable Guide Stars-drops to 0.44 of the nominal level. Moreover, almost all the lost portion of the celestial sphere is beyond $30^{\circ}$ from the galactic equator. An increase in the improvement in the Fine Lock guiding process to $V=13.5$ mag raises this probability to 0.60 . Thus, the rate at which sky coverage is regained is a slowly varying function

1 The Pointing Control System logic implemented by Lockheed Missiles Corp. discards threequarters of the photons acquired by the telescope. This egregious procedure reduces the effective limiting magnitude by $1.5 \mathrm{mag}$ from the one otherwise attainable. This represents a separate, additional problem not dealt with herein. 


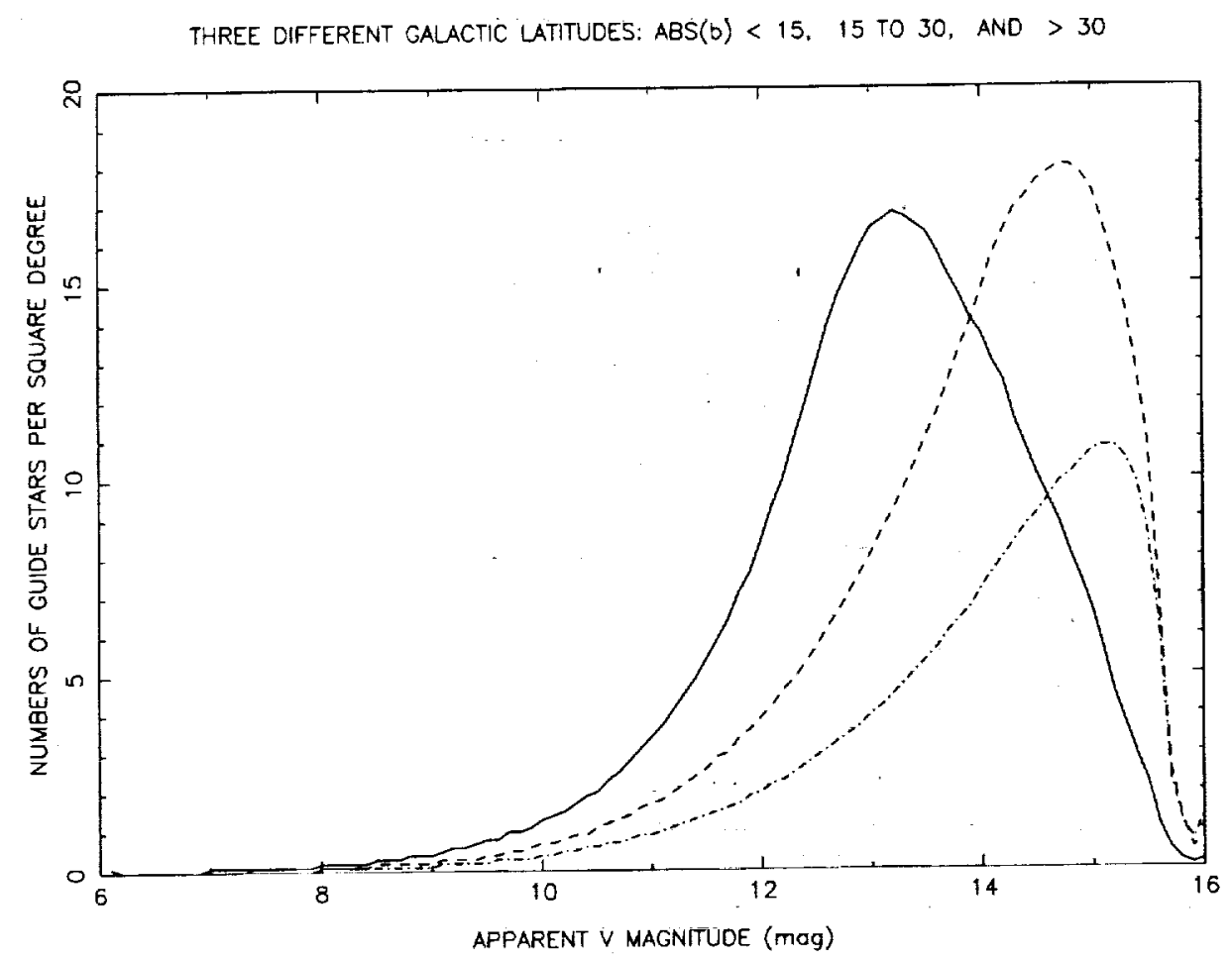

igure 1. Guide Star Catalog number distributions vs. apparent magnitude at three galactic latides.

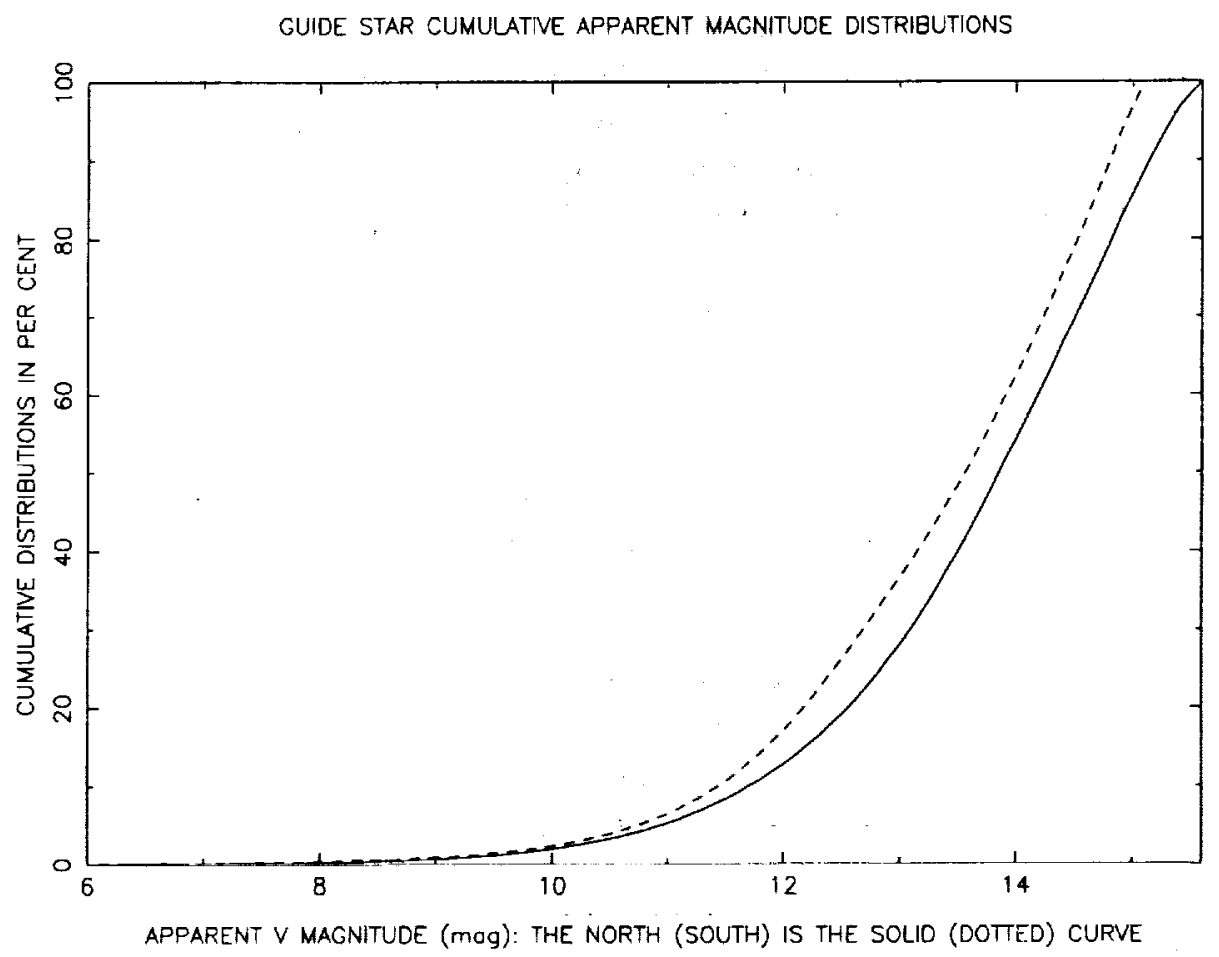

igure 2. Guide Star Catalog cumulative numbers vs. apparent magnitude. 
of the limiting magnitude primarily because the current limiting magnitude is so bright. Finall: retrieving $V=14.0 \mathrm{mag}$ performance, as I suggest can be routinely done, brings us back to $79 \% \mathrm{c}$ the $V=14.5 \mathrm{mag}$ level.

\section{THE DESIGN AND FUNCTIONING OF AN FGS}

\subsection{Optical System}

The optical train of a Fine Guidance Sensor (FGS) is displayed in Fig. 3 (see also Taff 1990b) The Optical Telescope Assembly (OTA) system of the HST is a Ritchey-Chretien Cassegrain design Before the prime focus of the OTA is a plane pickoff mirror which deflects light into the FGS. Th FGS total field-of-view is defined by this mirror. Light diverging from the pickoff mirror hits as off-axis aspheric mirror which nearly collimates the beam. A collimated beam is required for sensin: wavefront tilt at the Koester's prism (see below). The beam then travels to the first "Star Selector" known as Star Selector A. It rotates about its optical shaft encoder axis; the angle of rotation $i$ denoted by $\theta_{A}$.

A ray striking Star Selector A, parallel to its rotation axis, will be (nominally) deviated by $406 .:$ arc minutes. Star Selector A acts in concert with a second star selector, B (whose rotation angle is $\theta_{B}$ ). It too accomplishes a (nominal) 6.77 degree deviation. Together they move the $5^{\prime \prime} \times 5^{\prime}$ instantaneous field-of-view of an FGS about its total field-of-view.

In between the two star selectors is a five element corrector group; this corrector group is placec just before the first pupil and its function is to provide better collimation. Also, this refractive grour corrects for field curvature and astigmatism (which are characteristics of the Ritchey-Chretien desigr of the OTA). In addition, it corrects for design spherical aberration, coma, and the small amounts of astigmatism found in the collimating asphere. (The corrector group rotates with Star Selecto; $\mathrm{A}$ as one mechanical assembly.) The corrector group does not correct for the mis-shapen primary mirror nor does it correct for improper tilt or decenter of the secondary mirror nor for mechanica displacements of FGS optical components.

The polarizing beam splitter after the filter wheel divides the light into two equal intensity beams in mutually orthogonal directions. Each beam is also plane polarized. Two beams--hence twc Koester's prisms-are required since a Koester's prism only senses wavefront tilt in one axis. The light bundle is next incident onto the face of a Koester's prism. Within the prism it is divided by a dielectric beam splitter which performs a wavefront division of the incident ray. These two channels are denoted by $A$ and $B$. The dielectric coating retards the transmitted beam by a quarter of $a$ wavelength while the reflected light is unaffected.

Located beyond the Koester's prism is a set of duplex reimaging optics, one for the A and one for the B channels. The first part of each unit, the doublet, images the star onto the field stop. The lens/field stop assembly is located in the back focal plane of the doublet. The lens produces the pupil image on the sensitive surface of the photocathode tube. The $5^{\prime \prime}$ by $5^{\prime \prime}$ (object space) field stop provides the boundaries for the FGS instantaneous field-of-view. There is a photomultiplier tube for each channel of each Koester's prism, hence, four photomultiplier tubes reside in each FGS. The response of the photomultiplier tube is similar to that of the S-20 tube.

\subsection{The FGS Transfer Function and the Fine Error Signal}

\subsubsection{The Transfer Function}

Before discussing the algorithms contained in the Fine Guidance Electronics (FGE), it is important to describe the FGS Transfer Function. Figure 4 shows two situations. In the top picture there exists zero tilt in the wavefront at the face of the prism. That is, a combination of re-positioning the spacecraft and the Star Selectors has placed the target Guide Star onto the combined optical axis. Therefore, each photomultiplier tube for this prism senses the same amount of light. In the 


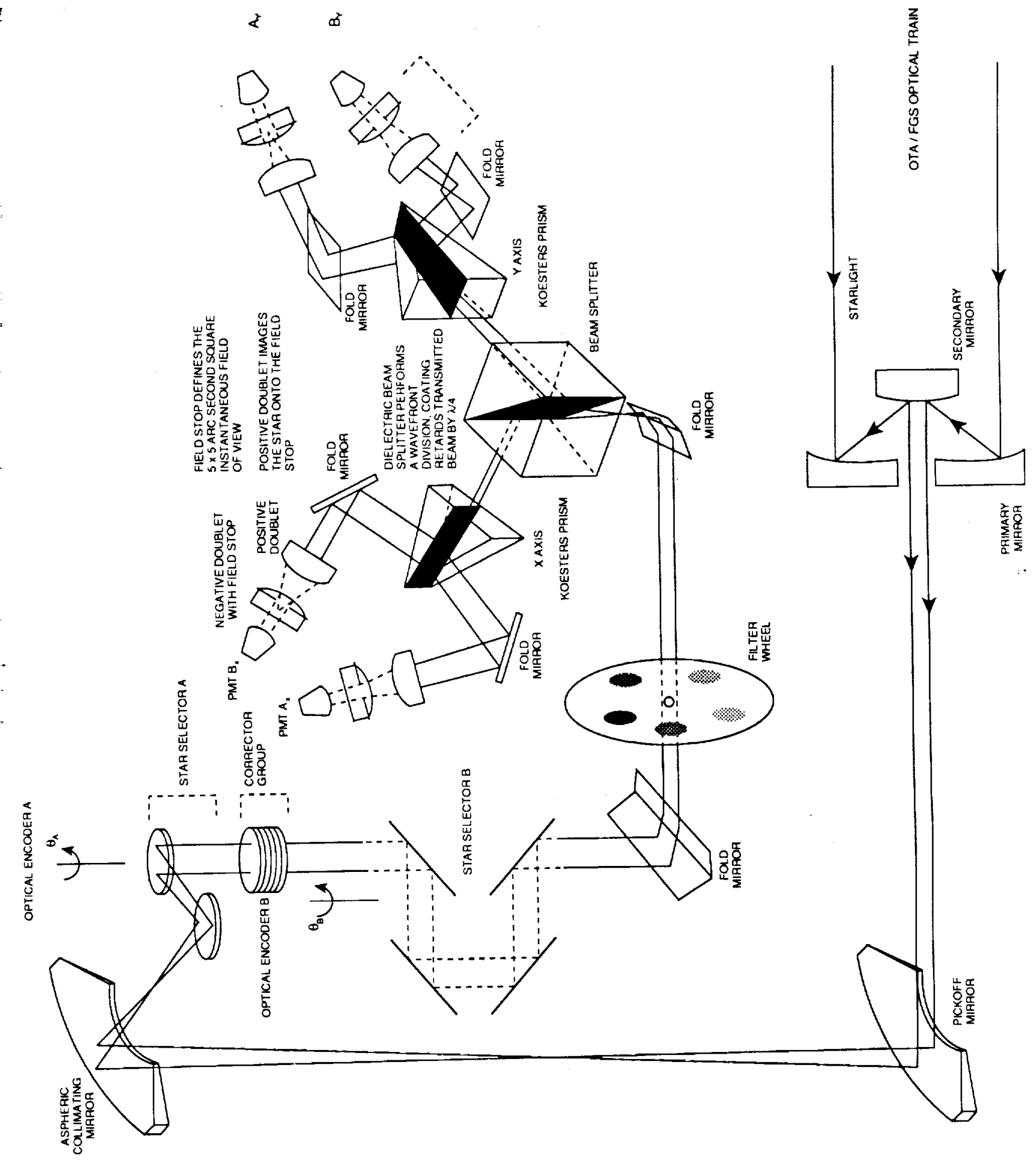

Figure 3. FGS Optical Train Schematic. 
lower part of the figure the wavefront has a quarter wave tilt. As the beam exits the left side of $t$ ] Koester's prism constructive interference occurs. (The wavefront which is transmitted through $t$ ] beam splitter is additionally retarded by $\lambda / 4$.) The right side will experience destructive interferenc Hence, the counts for the left side photomultiplier tube are greater than those in the right side ph tomultiplier tube. A graph of the counts versus tilt angle is known as the Transfer Function (TF See Fig. 5.

In more mathematical detail, for a monochromatic ray of light with angular frequency $\omega$, tl resultant of one component of its electromagnetic field will be of the form

$$
I \cos (\omega t-\phi)+I \cos \left(\omega t-\phi+\frac{\pi}{2}\right)=2 I \cos \frac{\pi}{4} \cos \left(\omega t-\phi+\frac{\pi}{4}\right)
$$

where $\phi$ is the initial phase. The resultant intensity is proportional to $2 I^{2}$. More generally, with sorr angle of incidence of the ray normal with respect to the Koester's prism face of $\theta$ at a distance $r$ or from the optical axis, the resultant component of the electromagnetic field has the form

$$
I \cos \left(\omega t-\phi+2 \pi \delta_{L, R} \theta r / \lambda\right)+I \cos \left(\omega t-\phi+2 \pi \delta_{L, R} \theta r / \lambda+\frac{\pi}{2}\right) .
$$

The wavelength associated with $\omega$ is $\lambda$ and $\delta_{L, R}$ is \pm 1 depending on whether the ray passed throug the lefthand or righthand side face of the Koester's prism. Ignoring the temporal modulation, th net energy is proportional to

$$
E_{L, R}=2 I^{2} \cos ^{2}\left(2 \pi \delta_{L, R} \theta r / \lambda+\frac{\pi}{4}\right)
$$

The FGE combines the counts from the A and B channels of the Koesters prism to form the TF (se Fig. 5). That is,

$$
S=\frac{A-B}{A+B}
$$

in which $A$ and $B$ are the counts in the $\mathrm{A}$ and $\mathrm{B}$ channels. In terms of $E_{L, R}, A$ and $B$ are proportioní; to

$$
e_{L, R}=\int_{0}^{R} 2 I^{2} \cos ^{2}\left(2 \pi \delta_{L, R} \theta r / \lambda+\frac{\pi}{4}\right) d r=\int_{0}^{R} E_{L, R} d r
$$

where $R$ is the radius of the primary mirror. Thus,

$$
S=\frac{e_{L}-e_{R}}{e_{L}+e_{R}}=\sin ^{2} z / z, \quad z=2 \pi \theta R / \lambda
$$

This is the Green's function for the FGS optical system and we may build up a theoretical TF b: integrating it over a hypothetical stellar spectrum, angular disc with limb darkening, actual photo multiplier responsivity, and so on (see Taff 1991).

\subsubsection{Fine Error Signal}

Once the FGS is locked onto a star, the Fine Error Signal is used to update the Star Selecto] positions so that the wavefront maintains zero tilt at the face of the Koester's prism. This proces: maintains the high precision pointing required for HST guiding. As can be seen from Fig. 5, the corc part of the TF is approximately linear from -10 to +10 mas. While the slope of the TF does change with stellar color index, this does not represent a significant variation.

The Fine Error Signal is defined in the FGE as

$$
R_{x}=K_{1 x} * S_{x}+K_{0 x}, \quad R_{y}=K_{1 y} * S_{y}+K_{0 y}
$$

where $S_{x}$ and $S_{y}$ are the FGE TFs for the $x$ and $y$ axes ala Eq. (1). $K_{1 x}$ and $K_{1 y}$ are the signal gains for each axis and are dependent upon stellar magnitude and background brightness [see Eq. (10)]. 


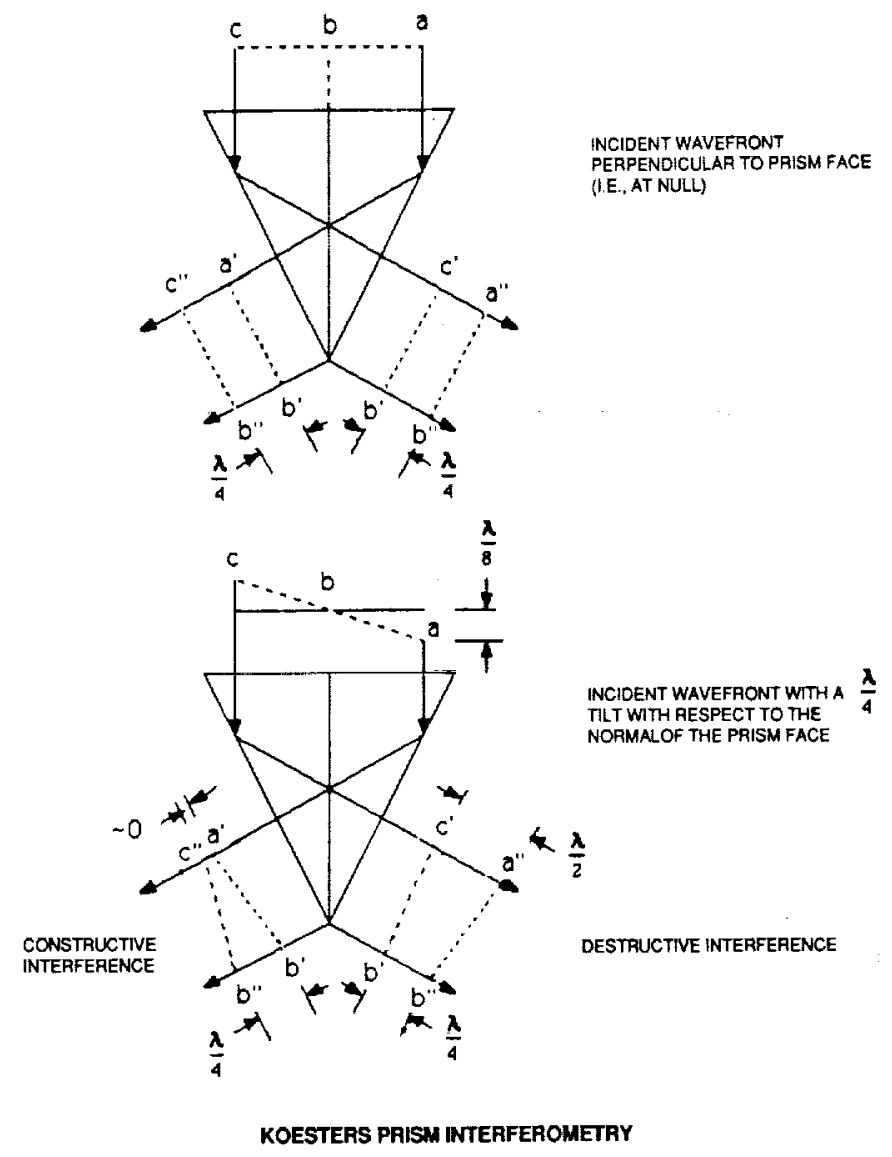

igure 4. Koester's prism interferometry without wavefront tilt (top) and with $\lambda / 4$ tilt (bottom).

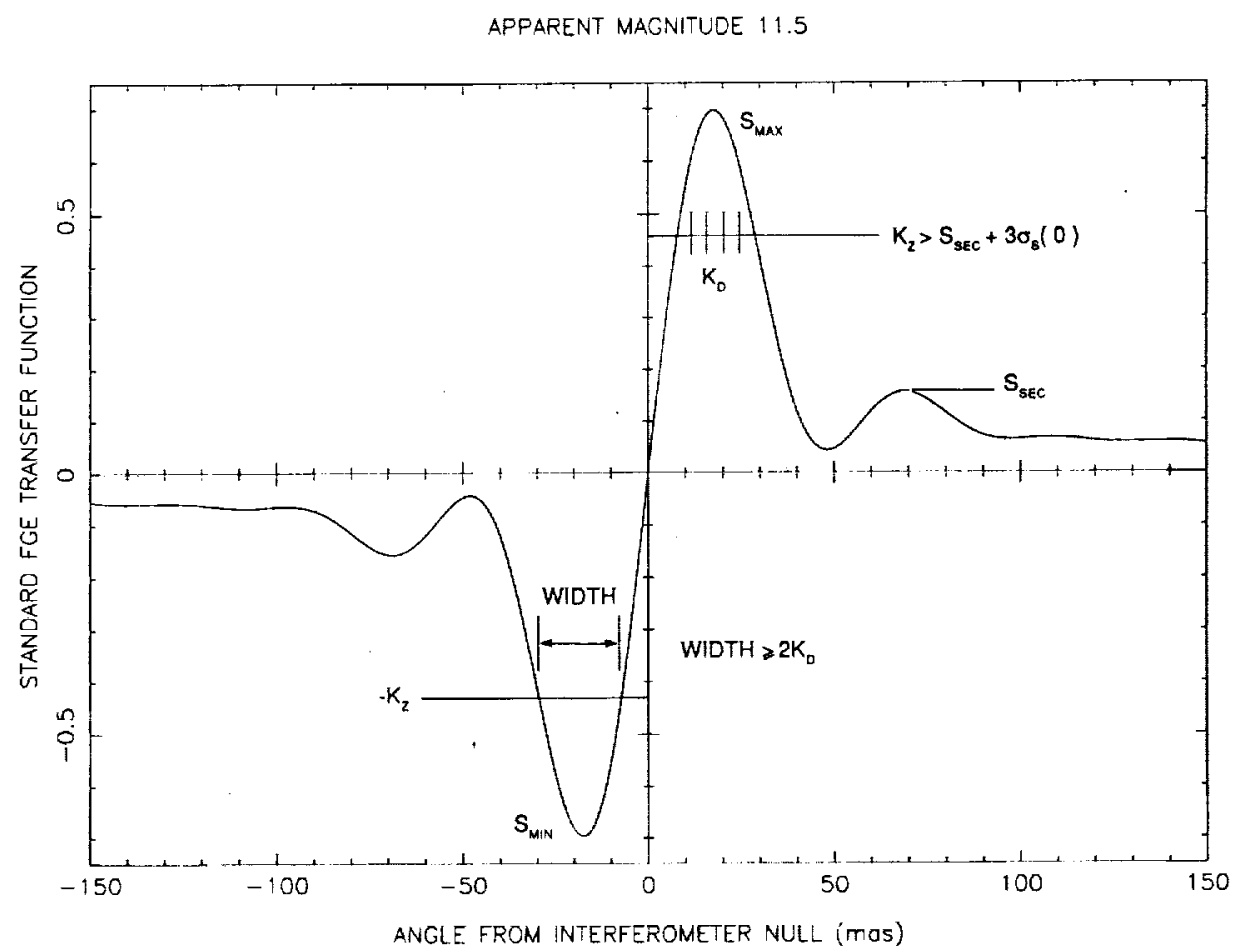

igure 5. Theoretical Transfer Function (TF). 
$K_{0 x}$ and $K_{0 y}$ are offsets whose initial purpose should be the equalization of the probability of a successful threshold detection whether one approaches the null of the interferometer from the left or the right [see Eq. (11)]. Values for these, and other, parameters can be changed via uplink telemetry.

The 'FGE Transfer Function' differs from that in Eq. (1) in two respects. First, to reduce sensitivity to photon noise, a mean value of $A+B$ is used to normalize $S$. This average is taken over the first sixteen samples after the Coarse Track phase has successfully terminated and just before the commencement of the 'walkdown' (the walkdown is described in §4.3). In addition, the mean, over these same 16 samples, of $A-B$ is computed, call it $\Delta_{A B}$, and subtracted from the numerator of $S$ in Eq. (1), as in Eq. (4) below. This adjusts for any $A$ vs. $B$ photomultiplier tube bias.

\section{FGS GUIDANCE OPERATION}

\subsection{Search Mode}

Search mode is entered into when the spacecraft's main computer issues a Search/Track "On" command to the Fine Guidance Electronics (FGE). The FGE will generate the appropriate Star Selector servo commands, at a 40 hertz rate, to move the $5^{\prime \prime} \times 5^{\prime \prime}$ instantaneous field-of-view of the FGS in an outward spiral (there is nominally a $30 \%$ overlap in coverage from one spiral line to the next). The purpose of Search mode is to search for a specific target (i.e., the Guide Star in this scenario). Success is based upon the photomultiplier tube count rate exceeding a lower limit threshold.

\subsection{Coarse Track Mode}

Once the target Guide Star has been detected in Search mode, the FGE will command the Star Selectors such that the instantaneous field-of-view will circle about the target at a once per second rate. The nominal nutation circle radius is 2.706 arc seconds; the number of nutations is variable. The FGE algorithm for Coarse Track updates the position of that center every 25 milliseconds nominally for 12 complete circuits (although only every fourth sample is accepted by the Pointing Control System; there is no integration). Coarse Track produces an error signal based on the combined photomultiplier tube counts it senses in each of the four quadrants of the nutation circle (see Taff 1990a for a fuller explanation). This signal then produces a new estimate for the center of nutation. The objectives of Coarse Track are to stabilize the still-drifting spacecraft (after a slew) and to determine the star's position to approximately 20 mas. Then a transition-stage, known as the 'walkdown', is used to reach the Fine Lock state.

\subsection{Fine Lock Mode}

The geometry of the approach to Fine Lock mode is shown in Fig. 6. The orthogonal intersection of the interferometers is commanded to a position $K_{B}$ arc seconds away from the target position (and midway in between them) which was determined in Coarse Track. Thus, approach can only occur along a diagonal and there is no provision for anisotropy (e.g., a $K_{b x}$ and a $K_{b y}$ ). The star selector encoders will be commanded by the FGE to approach the target position in at most $K_{5}$ steps, with each step being $K_{D}$ arc seconds in length (no $K_{d x}$ nor $K_{d y}$ ). The process of stepping down to the star colloquially referred to as the 'walkdown.'

The number of walkdown steps may vary up to 765 . The nominal walkdown step size was 0.009 arc seconds (it is now 6.5 mas). When the target Guide Star is "detected" in one of the interferometer axes, the step size for that axis is halved to prevent overshoot. Detection occurs when the interferometer signal exceeds a predetermined threshold ( $K_{Z}$; no $K_{z x}$ nor $K_{z y}$ ) for three consecutive 0.025 second samples (this is colloquially referred to as the 'three-hit algorithm'). Once a Guide Star is acquired, then the FGE control system will position the Star Selectors such that it will be simultaneously maintained in the linear region (at or near the null) of the interferometer axes (Sec. 3.2.2). 


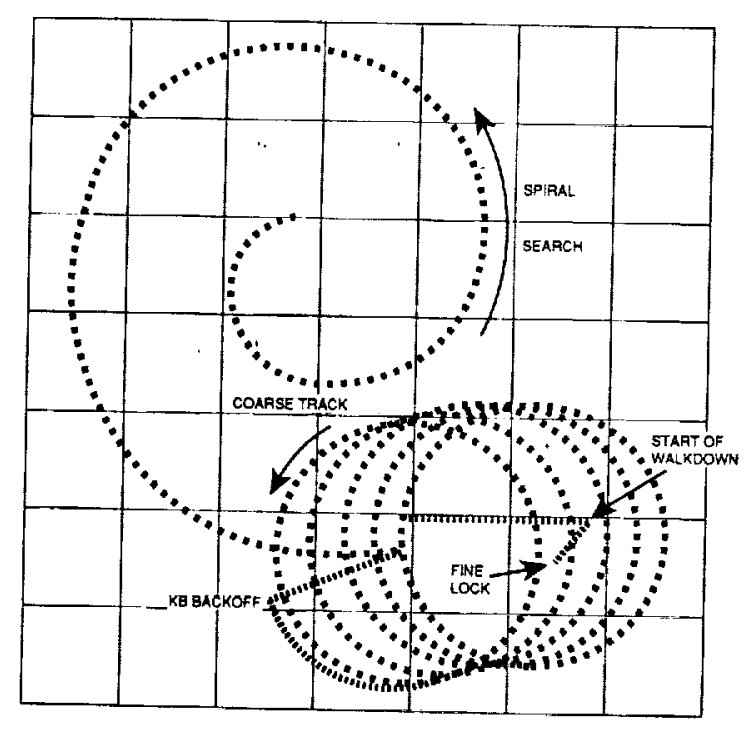

gure 6. Schematic Coarse Track/Fine Lock Process

\subsection{Discussion}

To obtain the Fine Lock state on both axes of the two orthogonal Koester's prisms there are 'en parameters available to us. (There should be at least two sets of seven for minimal flexibility.) e septuplet consists of $K_{5}$ (or $K_{B} ; K_{5} K_{D}=K_{B} / \sqrt{2}$ ), $K_{D}, K_{Z}, K_{1 x}$ and $K_{1 y}$, and $K_{0 x}$ and $y$. After defining these parameters and illustrating the role(s) they play in the Fine Lock process, rovide a theoretical analysis of maximizing the probability of reaching the Fine Lock state. Note it being in the Fine Lock state as far as the FGE is concerned refers to a successful passage through "'three-hit' algorithm. It does not necessarily say anything regarding the location of the Guide ir with respect to the optical axis. For instance, on a faint Guide Star we might 'lock' onto noise ih the star still far away from the interferometer null(s). (This has, in fact, happened with the real (Ss.) Whenever the 'three-hit' algorithm is satisfied, the Fine Lock condition, in the sense that the evant flag in the FGE is reset, is established.

Remembering the general discussion of FGS operations (for guidance) given above, after the arse Track state has been maintained for $K_{Y}(\sim 12)$ circuits of the nutation circle the position the photocenter has been reliably ascertained and the spacecraft drift stabilized. The FGE then nmands the star selector servos to place the star a certain distance away from the photocenter's ation (i.e., the backoff distance $K_{B}$ ) and at an orientation $45^{\circ}$ with respect to the interferometer is. (An orientation of $225^{\circ}$ is also possible.) Once at this point we commence the 'walkdown' 'cess towards the photocenter and, if we are successful at passing the 'three-hit' algorithm, an ntual Fine Lock state. $K_{5}$ is the maximum number of steps that can be taken on the 'walkdown'. must be large enough to ensure that we can pass through both the Transfer Function extrema. zause of the reduction of the step size once the threshold test has been successfully passed (by ${ }_{0}$, see the $K_{D}$ discussion below), $K_{5}$ must be larger than (total distance to the other side of the 
Transfer Function)/(step size/2). (Any bias between the Coarse Track photocenter position and th interferometer null positions should have been incorporated into the original offset from the Coars Track photocenter position, i.e., in $K_{B}$. Such a systematic difference could arise for a number c reasons not dealt with herein. These causes need not maintain-and indeed have not maintainedsymmetry with respect to the $x$ and $y$ axes. Therefore, there should have been provision for a $K_{\varepsilon}$ and a $K_{5 y}$ as well as a $K_{b x}$ and $K_{b y}$.)

You might wonder at the need for a $K_{5}$ at all. Given that the Coarse Track/Fine Lock offse vector has been well-determined (which is not yet the case at the beginning of 1991), we might adju: the Coarse Track backoff from the interferometer nulls to be between the two maxima (or minima fc the other polarity) of the TF. This is the place marked $S_{S E C}$ in Fig. 5. Even if this were done we sti need a maximum number of attempts at passing the 'three-hit' algorithm else we might never ex from the 'walkdown' state. Claiming that this is a Guide Star, and that therefore something is know regarding the star's surroundings and its apparent $V$ magnitude, does not vitiate the argument. $\mathrm{Tb}$ photometric precision of the Guide Star Catalog is only \pm 0.4 mag and there is a color term betwee the northern and southern celestial hemispheres [i.e., $J=V+0.72(B-V)]$. This has been adjuste for, on the average, by assuming that the typical Guide Star is a $\mathrm{K}$ or $\mathrm{M}$ dwarf (so $J \simeq V+0.6$ mag A less common spectral type will not be correctly handled with respect to the color index term.

The need for a parameter which fulfills the function of $K_{5}$ does not address the issue of why th 'walkdown' commences so far from the interferometer nulls. The alleged reason is that the TF ca not merely be $(A-B) /(A+B)$ for this does not incorporate different sensitivities or responsivitic between the photomultiplier tubes on the $A$ and $B$ channels. This bias, say $\Delta_{A B}$, is subtracted fror the difference term in the numerator so that

$$
S=\frac{A-B-\Delta_{A B}}{\langle A+B\rangle}
$$

(Remember that $S$ is normalized by the mean value of the first 16 samples, hence the angular bracket in the denominator.) $\Delta_{A B}$ is calculated at the start of the 'walkdown'. Thus, this position must $\mathrm{b}$ far enough from the interferometer nulls that the uncorrected value of the TF $[$ i.e., $(A-B) /(A+B$. would almost vanish were the two photomultiplier tubes perfectly matched. (Sixteen 0.025 sec sample are used to compute $\Delta_{A B}$.) By this mechanism the photomultiplier tube mismatch is always mad local-in time in case of aging of the tubes, in place on the celestial sphere in case of a variatio in the celestial background, and for this particular star in case it has an atypical color index. Eve more importantly, this procedure allows for the failure of one of the photomultiplier tubes withou destroying the capability of obtaining and maintaining Fine Lock. However, the real reason a larg $K_{B}$ is necessary is that the field stops in front of the photomultipliers have become displaced. Th net instantaneous field-of-view is the intersection of the four stops. This causes a significant Coars Track/Fine Lock bias, up to one arc second.

As we step towards the interferometer nulls from the Coarse Track backoff position, we do so wit steps of size $K_{D}$. (Perkin-Elmer Corp. originally used a value of 9 mas but it was since reduced, a a result of this analysis, to near the optimum of 6 mas.) The smaller $K_{D}$ is the longer it will take $t$ execute the 'walkdown', the larger $K_{5}$ must be, and the more danger there is that the position-to-rat converter, the piece of software in the FGE that actually computes the settings for the star selectc encoders, will stall. Remember that the star selectors are being commanded to move on the surfac of a sphere across a domain that has the shape of the FGS total field-of-view. Hence, the geometry $i$ non-planar and there is the possibility that a desired linear step will result in a very small projecte step. Since the position-to-rate converter is inhibited from taking very small steps-it has a 'leas significant bit criterion' of 3 bits (nominally; this is also adjustable) - too small a value of $K_{D}$ ma bring about this situation. Thus, the same portion of the TF will be repeatedly sampled. This $i$ actually a good thing to occur when we have a faint star and we are trying to satisfy the 'three-hit 
Igorithm. Since the step we take is halved whenever we pass the above-threshold query after not aving passed it during the last 25 milli-second photon integration period, the possibility of a stall icreases as we decrease $K_{D}$ just where, with respect to the TF, we want it to. Hence, the decision to wer $K_{D}$ from its initial value when the poor performance of the Fine Lock process became apparent, specially on fainter stars.

There are other bounds on $K_{D}$. In particular, the above-threshold portion of the TF must be t least $2 K_{D}$ wide and should preferably be $4 K_{D}$ across. Table 1 gives the two solutions to the juation $S(x)=K_{Z}$, for the $S>0$ portion of the curve (the theoretical curve has odd parity) for a ariety of values of $K_{Z}$ expressed as a percentage of the maximum value of the TF. To understand 1e reasoning behind the $2 K_{D}$ and $4 K_{D}$ lower bounds to the width of the above-threshold portion " the TF (see Fig. 5 again), consider the first step into the above-threshold portion of the curve om the right. The largest this advance could be is $K_{D}$. Suppose that this is the case and that e pass the $K_{Z}$ threshold test. Then the step size would be halved and we would penetrate $K_{D} / 2$ Irther. Suppose that once more the $K_{Z}$ threshold test is satisfied. We would take one more $K_{D} / 2$ ep, presumably pass the $K_{Z}$ threshold once again-now satisfying the 'three-hit' algorithm-and ien enter the null maintenance logic in the FGE. The total distance we traversed was $2 K_{D}$. More alistically we might want the width of the above-threshold portion of the TF to be large enough ; allow for one failing of the $K_{Z}$ test and still guarantee overall success at the 'three-hit' algorithm. 1 the worst circumstances this requires an additional minimum distance of $2 K_{D}$ whence the $4 K_{D}$ :alistic lower limit. For the nominal (i.e., Perkin-Elmer Corp.) threshold setting the width of the jove-threshold portion of the theoretical TF was 22.4 mas or just less than $2.5 K_{D}$ for a $K_{D}$ of 9 ias. Since we can assume that the entrance into the above-threshold portion of the TF is randomly id uniformly distributed, $4 K_{D}$ becomes $3.5 K_{D}$ in the mean or 21 mas $(=2.3$ the Perkin-Elmer orp. value of $K_{D}$ ).

Table 1. Fine Lock Values

\begin{tabular}{|c|c|c|c|c|}
\hline $\begin{array}{c}X_{L} \\
\text { (mas) }\end{array}$ & $\begin{array}{c}X_{U} \\
\text { (mas) }\end{array}$ & $K_{z} \cdot S_{M A X}$ & $K_{z}$ & $\begin{array}{l}\text { Width } \\
\text { (mas) }\end{array}$ \\
\hline 5.68 & 31.93 & 0.356 & 0.5 & 26.25 \\
\hline 6.99 & 29.92 & 0.427 & 0.6 & 22.93 \\
\hline 8.44 & 27.89 & 0.498 & 0.7 & 19.45 \\
\hline 10.12 & 25.71 & 0.570 & 0.8 & 15.59 \\
\hline 12.26 & 23.13 & 0.641 & 0.9 & 10.87 \\
\hline
\end{tabular}

In addition, in the presence of excessive spacecraft jitter, we want both $K_{D}$ and $K_{Z}$ to be as small : possible. The reason is, once the jitter per axis becomes comparable to $K_{D}$ itself we have too high probability of being thrown outside the above-threshold portion of the TF by a bodily movement of le spacecraft. Thus, we will (on the average) fail the $K_{Z}$ threshold test more often when the jitter larger. Within the FGE the only method we have of countering this is to maximize the number opportunities we can have to exceed the threshold. Lowering the threshold $K_{Z}$ widens the abovereshold portion of the curve and lowering $K_{D}$ maximizes the number of chances of testing against e threshold. Finally, if optical imperfections in the OTA or the FGSs cause the empirical TF to irrow with respect to the theoretical one, once again our only means of combating this within the $3 E$ is to reduce $K_{D}$. Of course neither spacecraft jitter nor optical defects have to be symmetrical ith respect to the faces of the Koester's prisms, so there should have been a provision for a $K_{d x}$ Id a $K_{d y}$. 
$K_{Z}$ is the much talked about threshold value. Since the most likely place to falsely declare the 'Fine Lock' state is when we traverse the secondary peaks in the TF (at the place marked $S_{S E C}$ in Fig. 5), the optimum value of $K_{Z}$ which will prevent this is $\left|S\left(X_{S E C}\right)\right|+3 \sigma_{S}(x=0)$. I have used the value of the standard deviation of the TF at the null because it is largest there. Such a three sigma criterion, built on the highest possible non-peak pedestal, ought to safely prevent a satisfaction of the 'three-hit' algorithm almost everywhere during the 'walkdown' process. Uncritically using this value can not be done because it gives no weight to how much of the above-threshold portion of the TF peak we will cut off. As discussed in detail above, the minimum width of the above-threshold portion of the TF is $3.5 K_{D}$. A simplified model of the photon-noise induced variation in the TF predicts that the standard deviation of the TF counts per axis from the star, the sky background, and the dark current noise in the photomultiplier tubes is $\approx 0.05$ hence, the lower limit to $K_{Z}$ can be safely met. Finally, because the imperfections in the OTA and the FGSs are not required to affect the $x$ and $y$ axis TFs in an identical manner, there should have been a provision for a $K_{z x}$ and a $K_{z y}$.

The remaining two $\mathrm{K}$-factors are used to adjust the instrumental $\mathrm{TF}$, given in Eq. (4), into one that will allow the FGE to succeed in the task of achieving the 'Fine Lock' state as per Eqs. (3). The first one I shall discuss removes any bias. Suppose that owing to optical imperfections, movements of the optical elements as a consequence of the exigencies of launch, deployment, or out-gassing, or : so on the positive peak of the instrumental TF is larger in magnitude than the negative peak of the instrumental TF on the same axis. Then, we would not have an equal chance of passing the 'three-hit' algorithm as we approached the interferometer null from the left and right sides. The purpose of $K_{0 x}$, and $K_{0 y}$ is to offset any such bias so that the TF the FGE has to deal with is symmetrical with respect to the probability of 'three-hit' algorithm passage. Thus, $S$ in Eq. (4) becomes

$$
S=\frac{A-B-\Delta_{A B}}{\langle A+B\rangle}+K_{0}
$$

Of course there is now a $S_{x}$ and a $S_{y}$ because there is provision for a $K_{0 x}$ and a $K_{0 y}$. The simplistic value of $K_{0}$ is clearly the peak-to-peak distance minus half the absolute value of one of the extrema, viz.

$$
K_{0}=\left[S_{M A X}-S_{M I N}\right]-S_{M A X} / 2 .
$$

This is the correct value for $K_{0}$ when we are trying to maintain the fine lock state with the Guide Star at the null of the interferometer. The reason is that this value of $K_{0}$ makes it equally difficult to climb over either extrema of the TF. This value for $K_{0}$ does not equalize the probability of success at the 'three-hit' altorithm [see $\S 5.1$, particularly just above Eq. (11)].

The two remaining $K$-factors, $K_{1 x}$ and $K_{1 y}$, unfortunately are forced to serve quadruple roles! Their first two functions are to correct the instrumental TF for the effect visible in Fig. 7. Because of the addition of the two channel photon counts in the denominator of $S$, as in Eq. (4) or (5), while they are subtracted in the numerator, the 'noise' component of the signal-namely the sky background and the dark current noise in the photomultiplier tubes-is compounded in the denominator but is eliminated in the numerator (on the average). Thus, as the Guide Star we are attempting to attain the 'Fine Lock' state on gets fainter, the instrumental TF naturally has a decreased fringe visibility. With $K_{z}$ fixed as a percentage of $S_{M A X}$, we may never pass the 'three-hit' algorithm for a fainter star. The initial purpose of $K_{1}$ is to boost the instrumental TF so that the $K_{z}$ threshold can be successfully passed even for fainter stars. Thus, $K_{1}$ must be a function of the apparent magnitude of the star as well as be different for each axis and each FGS. Whence, the final FGE version of the TF is given by

$$
Q=K_{1}\left\{\frac{A-B-\Delta_{A B}}{\langle A+B\rangle}\right\}+K_{0}
$$


APPAREVT MAGNITUDE VARIATIONS 11.5, 13.0, AND 14.5

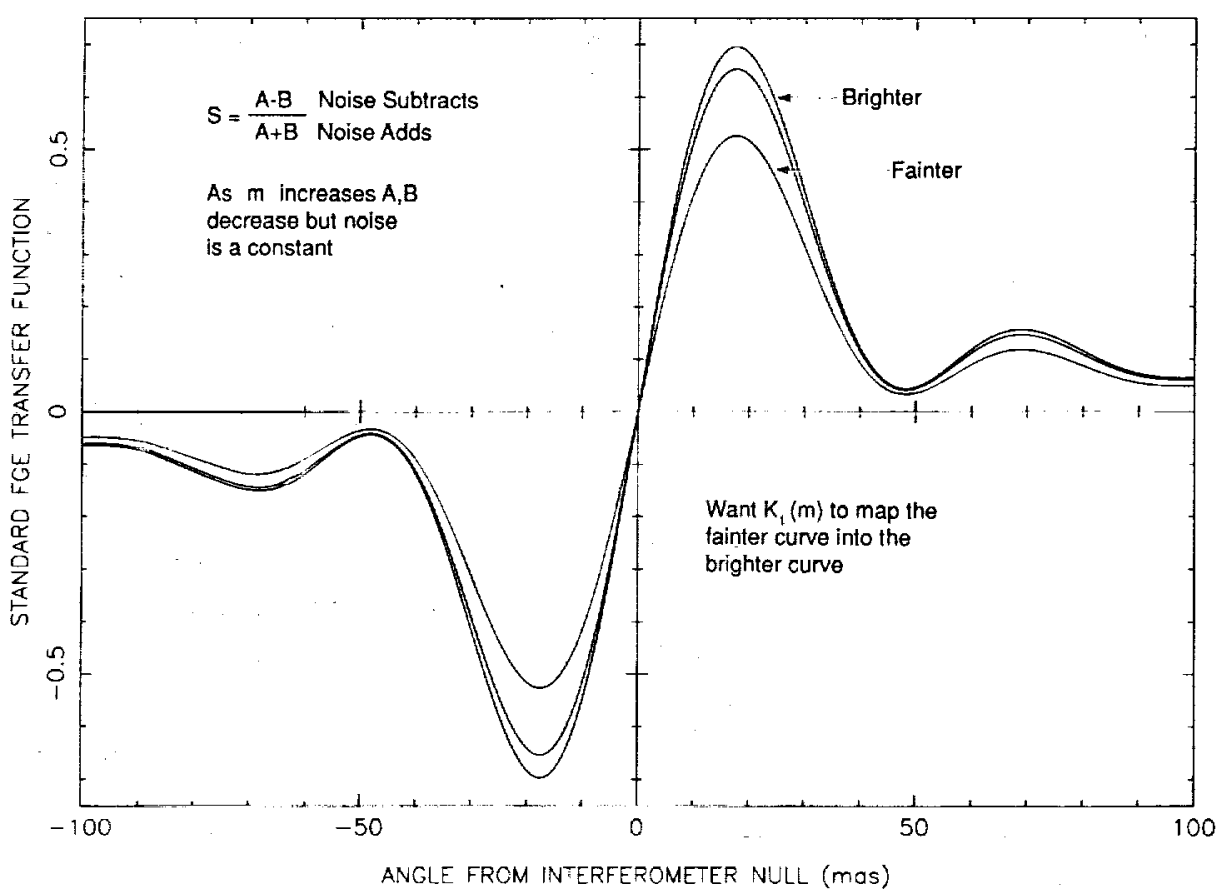

igure 7. Decrease in fringe visibility with apparent magnitude.

nowing what $K_{1}$ is supposed to do, it is easy to compute its value (although this is not the PerkinImer Corp. procedure). The other three roles of $K_{1}$ are more fully discussed in the next section. iriefly, $K_{1}$ is used during the walkdown to prevent a false lock, it is used in the Fine Lock state to revent a loss of lock, and it is simultaneously used in the Fine Lock state to minimize spacecraft ointing errors.

Finally, with definitions for $K_{0}$ and $K_{1}$ we can refine the constraint on $K_{Z}$ discussed above and 1own in Fig. 5. The real version of this is

$$
\left|K_{1}(m)\left[S_{S E C}+3 \sigma_{S}(0)\right]+K_{0}\right|<K_{Z}
$$

s prevent a false fine lock during the walkdown. Conversely, the constraint

$$
\left|K_{1}(m) K_{Z}+K_{0}\right|>K_{Z}
$$

lust also be satisfied else passing the "three-hit" algorithm will not occur. Perkin-Elmer Corp. lways uses a zero value for $K_{0}$ and $K_{1}(m)$ given by Eqs. (9) and (10) is always near 1.1, so these finements have little practical effect.

\section{ANALYSIS}

\subsection{Theory}

Let us start with the 'walkdown.' We need to be far away from the secondary maximum (miniJum; we shall assume that the polarity is such that we are approaching from the right in Fig. 5) of 1e TF in order for $\Delta_{A B}=\langle A-B\rangle$ to have meaning. From Fig. 5 any value of $K_{B} \gtrsim 200$ mas will 
do (i.e., less than half the Perkin-Elmer Corp. value). (Actually we can start as close as $K_{B}=50$ mas if we use $K_{0}$ to remove the bias.) Until we encounter the maximum of the TF we are only interested in not falsely locking. The place a false lock is most likely to occur is at the secondary maximum; hence, a threshold value of $K_{Z}$ in excess of $\left|S_{S E C}\right|+3 \sigma_{S}(0)$ is desirable.

Throughout, and especially during passage through the maximum (minimum), we want to correct for the effect in Fig. 7. The proper role of $K_{1}=K_{1}(m)$ then, is to map the fainter curve into the brighter curve. Specifically, imagine that we have a reference TF $S$ obtained on a very bright star of magnitude $m_{S}$. We can assume that we have used both ends of the empirical curve to obtain a statistically secure value for $\Delta_{A B}=\langle A-B\rangle$. Similarly we can use the entire curve to obtain a noise-free estimate of $C=\langle A+B\rangle$; whence

$$
S=\frac{A-B-\Delta_{A B}}{C} .
$$

Now $A$ is composed of both reference star photon counts $A_{S}$ and 'noise' counts. The latter arise from the sky background and the dark current noise. Symbolize the sum of the latter two components, which we can not easily separate, by $D$. Therefore,

$$
A=A_{S}+D, \quad B=B_{S}+D .
$$

Thus, on the average, we may recover $A_{S}$ and $B_{S}$ from Eq. (8),

$$
A_{S}=C(1+S) / 2+S D / 2, \quad B_{S}=C(1-S) / 2-S D / 2 .
$$

Now, for a Guide Star of apparent magnitude $m, A_{S}$ and $B_{S}$ become $\alpha$ and $\beta$ where

$$
\alpha=\kappa A_{S}, \quad \beta=\kappa B_{S} \text { with } \kappa=\operatorname{dex}\left[-0.4\left(m-m_{S}\right)\right] \text {. }
$$

Similarly $C_{S}=A_{S}+B_{S}$ becomes $\gamma=\kappa C_{S}$ and $S$ becomes $\sigma$,

$$
\sigma=\frac{\alpha-\beta}{\gamma+D}
$$

Clearly, then, the optimal value for $K_{1}(m)$ is just

$$
K_{1}(m) \sigma=S
$$

for this value of $K_{1}$ transforms the faint star TF into the (bright) reference star TF everywhere. The solution for $K_{1}$ may be written as

$$
K_{1}=1+D(1 / \kappa-1) / C \text {. }
$$

For real photomultiplier tube performance and realistic values of $C(\simeq 4$ per photomultiplier tube per $0.025 \mathrm{sec}$ ), $K_{1}$ ranges from 1.017 to 1.18 as $m$ ranges from 12.5 mag to 15.0 mag.

Before we leave the 'walkdown' and the possibility of false lock, if a real TF has a bias, then it has to be counteracted. Perkin-Elmer's theoretical value of $K_{0}$ was given in Eq. (6) and it only adjusts the extrema (in practice they always set $K_{0}$ equal to zero). As this value has to offer an equal probability of success at the 'three-hit' algorithm, this is too simplistic. In order to understand this I must digress a bit further.

Irregular bodily motions of the spacecraft, that is jitter, are deadly to the probability of success of the 'three-hit' algorithm. When compounded by too large a value of $K_{D}$, too high a value of $K_{Z}$, or an instrumentally narrowed set of above-threshold widths $W_{+}$and $W_{-}$, the problem becomes even more acute. ( $W_{ \pm}$are the linear widths of the above-threshold portions of the TF on the positive and 
(egative sides of the interferometer null.) Now, with a jitter amplitude $\gtrsim 1.5 K_{D}$ or $\gtrsim W_{+} / 2$ or $V_{-} / 2$, there is a reasonably high probability of being thrown out of the above-threshold portion of he TF even though the Star Selector encoders have been properly commanded to keep us within the esirable portion of the curve. Once on the shoulders of the extrema of the TFs the probability of assing the $K_{Z}$ threshold test is much diminished, hence no Fine Lock state.

One cure for this is to double our chances of achieving Fine Lock by attempting to pass the 'threeit' algorithm criteria on both extrema of the TF. [Indeed the real FGE accepts above-threshold rossings from both extrema sequentially (should this occur).] Hence, the desire to have both halves $f$ the curve be symmetrical with respect to this point. Clearly the sense in which they need to made ymmetrical is that the two widths be made equal; $W_{+}=W_{-}$and not that $S_{M A X}=\left|S_{M I N}\right|$. Thus, he implicit criterion defining $K_{0}$ is just

$$
W_{+}^{\prime}=W_{-} .
$$

So far we have fixed $K_{0}, K_{1}$, and $K_{B} . K_{D}$ should be as small as possible such that once it ; halved, it will not cause the Star Selector servos to stall $\left(K_{11}\right.$ determines the least significant it criterion in the position-to-rate converter). $K_{Z}$ must be determined by actually simulating the valkdown' and threshold-crossing process with an overall figure of merit, for the entire procedure, 1 mind. $K_{Z} \simeq 0.45$ is typically optimal.

This brings us to another essential point not considered by Perkin-Elmer Corp. In assessing the erformance of a complicated electro-optical system such as an FGS, especially in a situation wherein ze device is remotely located and it is (effectively) impossible to repair or alter it, the maximum mount of flexibility must be built in after a thorough analysis of the entire system's operation has een conducted. Such an analysis should include all of the obvious things which might go wrong 3 well as a few of the things which can not possibly (sic) go wrong. In the end, the non-linear ptimization has to be decided on by an overall figure of merit. None of this is evident in Perkinlmer Corp. documentation. As a specific example consider the Fine Lock process we have been iscussing. There are four different functions to successfully perform during the Fine Lock process: ) Avoid a false lock during the 'walkdown'; (ii) Achieve success at the 'three-hit' algorithm and o these two things reliably for $V \simeq 14.5 \mathrm{mag}$ stars; (iii) Once Fine Lock is achieved maintain a osition near the interferometer null, that is avoid a loss of lock; and (iv) Maximize the pointing istem's stability. Perkin-Elmer makes one value of $K_{1}$ try to perform all four of these things with no lought given to an overall optimization. (In fact, they choose $K_{1}$ solely to satisfy the no loss-of-lock iterion.)

\subsection{Numerical Experiments}

Late in 1990 a single bright star (Upgren $62, V=9.55 \mathrm{mag}$ ) was placed in nine different locations $\vdots$ each FGS field-of-view and five transfer scans obtained. From these nonets two curves per axis per GS were selected; one as "typical" and one as "unusual". For this set of a dozen single axis TFs a etailed simulation of the walkdown, three-hit algorithm, and null maintenance aspects of Guide Star zquisitions were simulated. Some generalities followed, to wit: (1) Spacecraft jitter, above 5 mas gr axis on a 0.025 second timescale, is deadly to three-hit algorithm satisfaction. Fortunately real sacecraft jitter does not have high frequency components of this amplitude. All further simulations ere performed with a real spacecraft jitter file rather than a $40 \mathrm{~Hz}$ Gaussian as previously. (2) ${ }_{B}$ needs to be as small as the Coarse Track/Fine Lock bias will allow. A larger value of $K_{B}$ just creases the probability of a lock on photon noise during the walkdown stage. (3) $K_{D}$ should be ist larger than twice the position-to-rate converter stall value. This greatly helps the probability success at the three-hit algorithm while only minimally extending the time interval necessary to zcomplish the walkdown stage. (4) $K_{0}=K_{0}\left(K_{Z}\right)$ is a complex function whose utilization has still st been thoroughly explored. (5) Real TFs are asymmetrical and the inability to have even a quartet 
of directional options will diminish the probability of fulfilling the scientific goals of the HST mission and (6) Large, rapidly fluctuating jitter is deadly to the maintenance of fine lock, especially for fainte: stars.

Before the large set of numerical experiments mentioned above were conducted, theoretical TFs such as those shown in Fig. 5, were used to test and debug the tripartite simulator software. Ever before I had real TFs and real jitter files, the above list of generalities was plainly evident. Thus only a very limited subset of all the results so far obtained are presented below.

Table 2 shows the results of a more focused set of numerical experiments ( $K_{D}=6$ mas, $K_{0}=0$ real spacecraft jitter). There are twelve sets of results in Table 2, four for each FGS. The origin $o$ : these quartets are two doublets, one for each axis (i.e., $x$ or $y$ ) in each FGS. Each component of the doublet is chosen from among the nine points in the aforementioned engineering test. After manua evaluation of the TFs at each of those nine places two were selected; one being close to the pre-launcl theoretical expectation and one being typically realistic for that axis in that FGS. The full FGF software simulator, with a real jitter file, was executed on each of the dozen curves and the various probabilities of success were computed. In no case, for reasonable values of the $K$ factors, was a loss of lock ever encountered for a (simulation) time duration of 10 minutes (i.e., $40 \times 60 \times 10$ executions of the null maintenance logic given a randomly placed start in between the extrema of the TF). Thus: the probability of a loss of lock, with this jitter file, is zero and will not be further discussed. A more indepth analysis of the jitter file shows it to be a little more quiet than is typical, hence it is stil premature to say that we know how to prevent a loss of lock during non-terminator crossing inducec disturbances. (One reason this jitter file was chosen was that it was a very long one-this selection effect biased it towards being unusually uneventful too.)

Table 2 contains the probability of success of the three-hit algorithm during threshold crossing $P_{t}$. the probability of success of the three hit-algorithm during the walkdown stage, $P_{w}$, and the overal! probability of success

$$
P_{t o t}=\left(1-P_{w}\right) * P_{t} *\left(1-P_{l o l}\right)
$$

where $P_{l o l}$ is the loss of lock probability. There are two rows per Guide Star apparent $V$ magnitude, one for the highest value of $P_{t o t}$ and one for the second highest value (as the $K$ factors were varied). By giving both of these one can evaluate the sensitivity of the optimal state with regard to perturbations: Each apparent magnitude also has two columns, one for each direction of approach that the FGE allows.

Perusing Table 2 one can rapidly conclude that guidance on fainter Guide Stars than we are currently using is eminently probable if one controls the FGS in a rationally determined manner.: There is also a marked asymmetry in some of the results so that the direction of approach is an: important variable and should not merely be left at the pre-launch (default) value. The asymmetry: in the TF is a non-linear combination of primary mirror misfiguring, secondary mirror misplacement (both in tilt and in decenter), and in individual FGS mechanical and optical defects. None of this, beyond the existence and magnitude of the primary mirror spherical aberration, is understood nor: capable of even being modeled (at the moment; the model I have suggested for the optical aberrations: Perkin-Elmer Corp. refuses to even numerically attempt). In particular, if one starts at the outer. edge of the FGS in Radial Bay \#1 and moves across its field-of-view towards the FGS in Radial Bay \#2 there is a continous change in the shape of the TFs which carries over to the next FGS, through its field-of-view, and then into the next FGS. This marked, field dependent, shape deformation can: only arise in the OTA and can not be spherical aberration!

What does the software simulator have to say about the current $\mathrm{K}$ factor settings? These results are in Table 3 along with the my best overall success probabilities for the same apparent magnitudes (remember that Table 3 includes a significantly reduced value of $K_{D}$ so that the probability of false lock during the walkdown stage is a little increased and that the probability of a successful lock- 
Table 2. FGE Simulator Results

\begin{tabular}{|c|c|c|c|c|c|c|c|c|c|c|}
\hline$V$ & $K_{Z}$ & $K_{1}$ & $\begin{array}{l}\text { Left } \\
P_{t}\end{array}$ & $P_{w}$ & $P_{t o t}$ & $K_{Z}$ & $K_{1}$ & $\begin{array}{l}\text { Right } \\
P_{t}\end{array}$ & $P_{w}$ & $P_{t o t}$ \\
\hline \multicolumn{11}{|l|}{$F G S 1-X 1$} \\
\hline \multirow[t]{2}{*}{13.25} & 0.45 & 0.90 & 100.0 & 0.0 & 100.0 & 0.55 & 0.90 & 99.5 & 0.5 & 99.0 \\
\hline & 0.55 & 1.00 & 99.5 & 0.0 & 99.5 & 0.55 & 1.00 & 99.5 & 1.0 & 98.5 \\
\hline \multirow[t]{2}{*}{14.00} & 0.55 & 0.80 & 93.0 & 3.5 & 89.7 & 0.65 & 1.00 & 95.5 & 3.5 & 92.1 \\
\hline & 0.65 & 1.00 & 92.5 & 4.5 & 88.3 & 0.55 & 0.80 & 92.0 & 2.5 & 89.7 \\
\hline \multirow[t]{2}{*}{14.75} & 0.65 & 0.80 & 79.5 & 16.5 & 66.4 & 0.65 & 0.80 & 68.0 & 28.0 & 49.0 \\
\hline & 0.65 & 0.90 & 87.5 & 36.5 & 55.6 & 0.75 & 0.90 & 63.0 & 27.0 & 46.0 \\
\hline \multicolumn{11}{|l|}{$F G S 1-X 2$} \\
\hline \multirow[t]{2}{*}{13.25} & 0.65 & 1.00 & 98.0 & 2.5 & 95.6 & 0.65 & 1.00 & 99.5 & 7.5 & 92.0 \\
\hline & 0.55 & 0.90 & 100.0 & 4.5 & 95.5 & 0.65 & 0.90 & 94.5 & 3.0 & 91.7 \\
\hline \multirow[t]{2}{*}{14.00} & 0.65 & 0.80 & 84.5 & 8.0 & 77.7 & 0.65 & 0.80 & 82.5 & 19.0 & 66.8 \\
\hline & 0.75 & 0.90 & 76.5 & 7.0 & 71.1 & 0.65 & 0.90 & 95.5 & 32.0 & 64.9 \\
\hline \multirow[t]{2}{*}{14.75} & 0.75 & 0.80 & 53.0 & 38.5 & 32.6 & 0.75 & 0.80 & 61.0 & 44.0 & 34.2 \\
\hline & 0.65 & 0.80 & 78.0 & 67.5 & 25.4 & 0.75 & 0.90 & 75.0 & 74.0 & 19.5 \\
\hline \multicolumn{11}{|l|}{$F G S 1-Y 1$} \\
\hline \multirow[t]{2}{*}{13.25} & 0.45 & 0.80 & 100.0 & 0.0 & 100.0 & 0.45 & 0.80 & 100.0 & 0.0 & 100.0 \\
\hline & 0.55 & 0.80 & 100.0 & 0.0 & 100.0 & 0.45 & 0.90 & 100.0 & 0.0 & 100.0 \\
\hline \multirow[t]{2}{*}{14.00} & 0.55 & 0.90 & 100.0 & 1.5 & 98.5 & 0.55 & 0.80 & 99.5 & 1.0 & 98.5 \\
\hline & 0.65 & 1.00 & 98.5 & 0.5 & 98.0 & 0.55 & 0.80 & 98.0 & 0.5 & 97.5 \\
\hline \multirow[t]{2}{*}{14.75} & 0.65 & 0.90 & 90.0 & 10.5 & 80.6 & 0.65 & 0.90 & 93.0 & 9.0 & 84.6 \\
\hline & 0.65 & 0.80 & 80.0 & 1.0 & 79.2 & 0.75 & 1.00 & 89.0 & 9.0 & 81.0 \\
\hline \multicolumn{11}{|l|}{$F G S 1-Y 2$} \\
\hline \multirow[t]{2}{*}{13.25} & 0.45 & 0.80 & 100.0 & 0.0 & 100.0 & 0.45 & 0.80 & 100.0 & 0.0 & 100.0 \\
\hline & 0.55 & 1.00 & 100.0 & 0.0 & 100.0 & 0.55 & 1.00 & 100.0 & 0.0 & 100.0 \\
\hline \multirow[t]{2}{*}{14.00} & 0.55 & 0.80 & 97.0 & 0.0 & 97.0 & 0.55 & 0.90 & 100.0 & 3.0 & 97.0 \\
\hline & 0.65 & 1.00 & 97.5 & 1.5 & 96.0 & 0.65 & 1.00 & 96.5 & 0.0 & 96.5 \\
\hline \multirow[t]{2}{*}{14.75} & 0.55 & 0.80 & 95.0 & 17.5 & 78.4 & 0.65 & 0.90 & 87.5 & 33.0 & 58.6 \\
\hline & 0.75 & 1.00 & 80.5 & 7.5 & 74.5 & 0.75 & 1.00 & 77.5 & 24.5 & 58.5 \\
\hline \multicolumn{11}{|l|}{$F G S 2-X 1$} \\
\hline \multirow[t]{2}{*}{13.25} & 0.55 & 0.80 & 93.5 & 93.0 & 6.5 & 0.55 & 0.80 & 94.0 & 85.0 & 14.1 \\
\hline & 0.45 & 0.80 & 98.5 & 98.5 & 1.5 & 0.55 & 0.90 & 99.5 & 97.5 & 2.5 \\
\hline \multirow[t]{2}{*}{14.00} & 0.55 & 0.80 & 93.5 & 99.5 & 0.5 & 0.45 & 0.80 & 100.0 & 100.0 & 0.0 \\
\hline & 0.45 & 0.80 & 100.0 & 100.0 & 0.0 & 0.45 & 0.90 & 100.0 & 100.0 & 0.0 \\
\hline \multirow[t]{2}{*}{14.75} & 0.45 & 0.80 & 100.0 & 100.0 & 0.0 & 0.45 & 0.80 & 100.0 & 100.0 & 0.0 \\
\hline & 0.55 & 0.80 & 99.5 & 100.0 & 0.0 & 0.55 & 0.80 & 100.0 & 100.0 & 0.0 \\
\hline
\end{tabular}


Table 2. Continued

\begin{tabular}{|c|c|c|c|c|c|c|c|c|c|c|}
\hline$V$ & $K_{Z}$ & $K_{1}$ & $\begin{array}{l}\text { Left } \\
P_{t}\end{array}$ & $P_{w}$ & $P_{t o t}$ & $K_{Z}$ & $K_{1}$ & $\begin{array}{l}\text { Right } \\
P_{t}\end{array}$ & $P_{w}$ & $P_{t o t}$ \\
\hline \multicolumn{11}{|l|}{$F G S 2-X 2$} \\
\hline \multirow[t]{2}{*}{13.25} & 0.55 & 0.80 & 98.0 & 1.5 & 96.5 & 0.55 & 0.80 & 99.5 & 1.5 & 98.0 \\
\hline & 0.65 & 1.00 & 98.0 & 2.0 & 96.0 & 0.65 & 1.00 & 100.0 & 4.0 & 96.0 \\
\hline \multirow[t]{2}{*}{14.00} & 0.65 & 0.80 & 85.0 & 10.5 & 76.1 & 0.65 & 0.80 & 83.5 & 17.0 & 69.3 \\
\hline & 0.65 & 0.90 & 93.0 & 38.0 & 57.7 & 0.65 & 0.90 & 90.0 & 34.5 & 59.0 \\
\hline \multirow[t]{2}{*}{14.75} & 0.65 & 0.80 & 85.5 & 74.5 & 21.8 & 0.65 & 0.80 & 76.5 & 70.0 & 23.0 \\
\hline & 0.65 & 0.90 & 91.5 & 91.5 & 7.8 & 0.65 & 0.90 & 84.0 & 90.0 & 8.4 \\
\hline \multicolumn{11}{|l|}{$F G S 2-Y 1$} \\
\hline \multirow[t]{2}{*}{13.25} & 0.65 & 0.80 & 91.5 & 1.0 & 90.6 & 0.65 & 0.80 & 89.0 & 7.0 & 82.8 \\
\hline & 0.65 & 0.90 & 94.5 & 6.0 & 88.8 & 0.65 & 0.90 & 98.5 & 16.0 & 82.7 \\
\hline \multirow[t]{2}{*}{14.00} & 0.75 & 0.80 & 72.0 & 11.5 & 63.7 & 0.65 & 0.80 & 90.0 & 40.5 & 53.6 \\
\hline & 0.65 & 0.80 & 87.5 & 32.5 & 59.1 & 0.75 & 0.80 & 64.0 & 21.5 & 50.2 \\
\hline \multirow[t]{2}{*}{14.75} & 0.75 & 0.80 & 64.0 & 83.5 & 10.6 & 0.75 & 0.80 & 63.5 & 89.5 & 6.7 \\
\hline & 0.75 & 0.90 & 78.5 & 96.5 & 2.7 & 0.65 & 0.80 & 85.0 & 97.0 & 2.6 \\
\hline
\end{tabular}

FGS2-Y2

$\left.\begin{array}{rrrrrrrrrrr}13.25 & 0.55 & 0.80 & 95.0 & 17.0 & 78.9 & 0.65 & 0.80 & 81.0 & 9.0 & 73.7 \\ & 0.75 & 1.00 & 85.5 & 8.0 & 78.7 & 0.65 & 0.90 & 89.0 & 24.5 & 67.2 \\ 14.00 & 0.75 & 0.80 & 61.0 & 29.0 & 43.3 & 0.75 & 0.80 & 61.5 & 25.5 & 45.8 \\ & 0.75 & 0.90 & 75.0 & 52.5 & 35.6 & 0.75 & 0.90 & 73.5 & 38.0 & 45.6 \\ 14.75 & 0.75 & 0.80 & 68.0 & 88.0 & 8.2 & 0.75 & 0.80 & 63.0 & 77.5 & 14.2 \\ & 0.75 & 0.90 & 78.0 & 95.5 & 3.5 & 0.75 & 0.90 & 68.0 & 95.5 & 3.1\end{array}\right)$

$\begin{array}{crrrrrrrrrr}\text { FGS3-X1 } & & & & & & & & & & \\ 13.25 & 0.55 & 0.90 & 100.0 & 0.0 & 100.0 & 0.45 & 0.80 & 100.0 & 0.0 & 100.0 \\ & 0.55 & 0.80 & 99.5 & 0.0 & 99.5 & 0.55 & 0.80 & 99.5 & 0.0 & 99.5 \\ 14.00 & 0.55 & 0.80 & 98.0 & 6.5 & 91.6 & 0.65 & 0.90 & 93.0 & 2.0 & 91.1 \\ & 0.65 & 0.90 & 93.5 & 2.0 & 91.6 & 0.55 & 0.80 & 97.5 & 8.0 & 89.7 \\ 14.75 & 0.75 & 0.90 & 75.5 & 14.0 & 64.9 & 0.65 & 0.80 & 88.5 & 24.5 & 66.8 \\ & 0.65 & 0.80 & 75.0 & 14.5 & 64.1 & 0.75 & 0.90 & 82.5 & 20.0 & 66.0\end{array}$

FGS3-X2

\begin{tabular}{rrrrrrrrrrr}
13.25 & 0.45 & 0.80 & 100.0 & 0.0 & 100.0 & 0.55 & 0.80 & 100.0 & 0.5 & 99.5 \\
& 0.45 & 0.90 & 100.0 & 0.0 & 100.0 & 0.65 & 1.00 & 99.5 & 0.0 & 99.5 \\
14.00 & 0.55 & 0.80 & 98.0 & 2.0 & 96.0 & 0.55 & 0.80 & 97.5 & 2.0 & 95.6 \\
& 0.65 & 0.90 & 97.0 & 1.5 & 95.5 & 0.65 & 1.00 & 98.0 & 3.0 & 95.1 \\
14.75 & 0.65 & 0.80 & 76.5 & 15.0 & 65.0 & 0.65 & 0.80 & 86.0 & 6.0 & 80.8 \\
& 0.65 & 0.90 & 86.0 & 29.0 & 61.1 & 0.65 & 0.90 & 90.5 & 14.0 & 77.8 \\
\hline
\end{tabular}


Table 2. Continued

\begin{tabular}{|c|c|c|c|c|c|c|c|c|c|c|}
\hline$V$ & $K_{Z}$ & $K_{1}$ & $\begin{array}{l}\text { Left } \\
P_{t}\end{array}$ & $P_{w}$ & $P_{t o t}$ & $K_{Z}$ & $K_{1}$ & $\begin{array}{c}\text { Right } \\
P_{t}\end{array}$ & $P_{w}$ & $P_{t o t}$ \\
\hline \multicolumn{11}{|l|}{${ }^{7} G S 3-Y 1$} \\
\hline \multirow{2}{*}{13.25} & 0.45 & 0.80 & 99.5 & 0.0 & 99.5 & 0.45 & 0.90 & 100.0 & 0.5 & 99.5 \\
\hline & 0.65 & 1.10 & 98.5 & 0.0 & 98.5 & 0.45 & 0.80 & 99.5 & 0.5 & 99.0 \\
\hline \multirow{2}{*}{14.00} & 0.55 & 0.90 & 95.0 & 6.5 & 88.8 & 0.45 & 0.80 & 98.0 & 14.0 & 84.3 \\
\hline & 0.65 & 1.10 & 96.0 & 7.5 & 88.8 & 0.55 & 0.90 & 89.0 & 7.5 & 82.3 \\
\hline \multirow[t]{2}{*}{14.75} & 0.55 & 0.80 & 77.0 & 29.5 & 54.3 & 0.55 & 0.80 & 75.0 & 28.5 & 53.6 \\
\hline & 0.65 & 0.90 & 72.0 & 22.5 & 55.8 & 0.55 & 0.90 & 80.0 & 50.0 & 40.0 \\
\hline \multicolumn{11}{|l|}{$\neg G S 3-Y 2$} \\
\hline \multirow[t]{2}{*}{13.25} & 0.45 & 0.80 & 100.0 & 0.0 & 100.0 & 0.45 & 0.80 & 100.0 & 0.0 & 100.0 \\
\hline & 0.55 & 0.90 & 100.0 & 0.0 & 100.0 & 0.45 & 0.90 & 100.0 & 0.0 & 100.0 \\
\hline \multirow[t]{2}{*}{14.00} & 0.65 & 1.20 & 100.0 & 0.0 & 100.0 & 0.55 & 0.90 & 99.0 & 1.0 & 98.0 \\
\hline & 0.75 & 1.20 & 100.0 & 0.0 & 100.0 & 0.55 & 1.00 & 99.5 & 1.5 & 98.0 \\
\hline \multirow[t]{2}{*}{14.75} & 0.55 & 0.80 & 94.0 & 7.5 & 90.0 & 0.55 & 0.80 & 90.5 & 18.5 & 73.8 \\
\hline & 0.65 & 1.00 & 93.0 & 8.0 & 85.6 & 0.65 & 0.90 & 84.5 & 10.0 & 76.1 \\
\hline
\end{tabular}

luring the threshold crossing stage is dramatically increased). Two things stand out in Table 3; 'erkin-Elmer Corp.'s $K_{1}$ is much too large and, as a consequence, their probability of false lock luring the walkdown often reaches certainty. The real cause of this is that the TFs which led to he $P_{w}=100 \%$ values are double humped; they are so deformed that to speak of a maximum and econdary maximum, as illustrated in Fig. 5 is very misleading. The height of the 'secondary' peak is ometimes more than half that of the primary. Thus, when an excesssively large value of $K_{1}$ is used, $t$ becomes almost a certainty that lock will occur on the secondary peak (as long as the direction if approach is such that this must be encountered; since it is rare to have both secondary extrema rronounced, this is another reason to vary the direction of approach with each FGS). Remember too hat the $K_{1}$ values in Table 3 are real whereas those in Table 2 are relative to the nominal value of $\zeta_{1}$ given by Eq. (10). As the nominal value is typically 1.1, Perkin-Elmer Corp.'s values of $K_{1}$ are j-3 times larger than the ones I would suggest.

This has severe operational consequences. First of all, when viewed as a successful or unsuccessful xck it gets counted as a successful one. It is rarely a stable one because jitter has a much easier ob driving the FGE over the secondary hump. When this happens we are so far away from the rue null that automatic recovery will almost never succeed. Similar remarks apply to a photonloise induced loss of lock from the secondary null. A second negative consequence of locking on he secondary peak is that the telescope is mis-pointed by the distance between the two nulls. This $s$ fatal to astrometry and fairly important for general target acquisition since the FGS to Science nstrument aperture alignment will be thrown off. This can easily amount to 50 mas. All of the bove, easily predictable, consequences have frequently occurred with the real spacecraft. Now there s a straightforward, unified, explanation for them. 
Table 3. FGE P-E Simulator Results

\begin{tabular}{ccrrrrr}
\hline \hline & & & & & \multicolumn{1}{c}{ LGT } \\
\hline 13.00 & 0.42 & 1.86 & 100.0 & 15.5 & 84.5 & 99.0 \\
14.00 & 0.42 & 2.04 & 97.5 & 71.5 & 27.8 & 92.2 \\
13.00 & 0.42 & 1.86 & 0.0 & 100.0 & 0.0 & 91.7 \\
14.00 & 0.42 & 2.04 & 0.0 & 100.0 & 0.0 & 66.8 \\
13.00 & 0.42 & 1.56 & 100.0 & 1.5 & 98.5 & 100.0 \\
14.00 & 0.42 & 1.62 & 100.0 & 17.0 & 83.0 & 100.0 \\
13.00 & 0.42 & 1.56 & 100.0 & 2.0 & 98.0 & 100.0 \\
14.00 & 0.42 & 1.62 & 97.0 & 18.0 & 79.5 & 97.0 \\
13.00 & 0.42 & 1.98 & 0.0 & 100.0 & 0.0 & 14.1 \\
14.00 & 0.42 & 2.22 & 0.0 & 100.0 & 0.0 & 0.0 \\
13.00 & 0.42 & 1.98 & 0.0 & 100.0 & 0.0 & 98.0 \\
14.00 & 0.42 & 2.22 & 0.0 & 100.0 & 0.0 & 69.3 \\
13.00 & 0.42 & 2.46 & 0.0 & 100.0 & 0.0 & 82.3 \\
14.00 & 0.42 & 2.70 & 0.0 & 100.0 & 0.0 & 53.6 \\
13.00 & 0.42 & 2.46 & 0.0 & 100.0 & 0.0 & 73.7 \\
14.00 & 0.42 & 2.70 & 0.0 & 100.0 & 0.0 & 45.8 \\
13.00 & 0.42 & 1.50 & 97.5 & 3.5 & 94.0 & 100.0 \\
14.00 & 0.42 & 1.68 & 90.5 & 24.5 & 68.3 & 91.1 \\
13.00 & 0.42 & 1.50 & 0.0 & 100.0 & 0.0 & 99.5 \\
14.00 & 0.42 & 1.68 & 0.0 & 100.0 & 0.0 & 95.6 \\
13.00 & 0.42 & 1.62 & 11.5 & 2.5 & 11.2 & 99.0 \\
14.00 & 0.42 & 1.74 & 13.0 & 21.5 & 10.2 & 82.3 \\
13.00 & 0.42 & 1.62 & 100.0 & 1.5 & 98.5 & 100.0 \\
14.00 & 0.42 & 1.74 & 99.5 & 32.0 & 67.7 & 98.0 \\
\hline & & & & & &
\end{tabular}

\section{REFERENCES}

L. G. Taff, "An Analysis of the Hubble Space Telescope Fine Guidance Sensor Coarse Track Mode," Exp. Astr., vol. 1, pp. 237-226, 1990a.

L. G. Taff, "The Astrometric Calibration of the Hubble Space Telescope Fine Guidance Sensors," Astrophys. J., vol. 365, pp. 407-418, Dec. 1990b.

L. G. Taff, "Expanding the Scientific Role of the Hubble Space Telescope Fine Guidance Sensors," in press in Adv. Space Res., 1991. 